\title{
Coupling characteristics of surface modes in truncated two-dimensional photonic crystals
}

\author{
Hyoung-Gyu Choi, Sang Soon Oh, Sun-Gu Lee, Myeong-Woo Kim, Jae-Eun Kim, and \\ Hae Yong Park ${ }^{\text {a) }}$ \\ Department of Physics, Korea Advanced Institute of Science and Technology, Daejeon 305-701, Korea \\ Chul-Sik Kee ${ }^{\text {b) }}$ \\ Nanophotonics Laboratory, Advanced Photonics Research Institute, GIST, Gwangju 500-712, Korea
}

(Received 1 October 2006; accepted 7 October 2006; published online 22 December 2006)

\begin{abstract}
We investigate the coupling properties of localized modes between two identical truncated triangular photonic crystals composed of air holes in a dielectric background. The frequency of even parity modes varies more sensitively to the distance between the truncated faces of the crystals; i.e., the coupling distance. This behavior is explained in terms of photonic bands and spatial distribution of fields. This localized mode with even parity is shown to be a good guided mode from the finite-difference time-domain simulation. The guided range can be tuned by varying the coupling distance and the degree of truncation. (c) 2006 American Institute of Physics.
\end{abstract}

[DOI: $10.1063 / 1.2401282$ ]

Photonic crystals (PCs) are optical materials with periodic changes in dielectric constant. ${ }^{1}$ It is well known that these structures can have photonic band gaps; that is, the frequency ranges where photons cannot propagate in any direction. Moreover, introducing various defects into the PCs can give rise to localized modes within band gaps and realize various applications such as control of spontaneous emission, zero-threshold lasing, optical filters, ${ }^{2}$ and ultrasmall optical circuits. ${ }^{3}$ Since all of the practical PCs necessarily have surfaces, that is, interfaces between PCs and the air, it is important to study the characteristics of surface modes for applications. ${ }^{4-10}$ Because surface modes act as one of the origins of the propagation loss, it is generally believed that these have undesirable features of finite PCs unlike point or line defect modes. ${ }^{11,12}$ Recently, however, remarkable results about the application of surface modes, which can enhance the coupling of light into and out of a PC waveguide and can become a lasing mode under pulsed optical pumping conditions, have been reported. ${ }^{13-15}$

In this article, we investigate the coupling characteristics of surface modes of two-dimensional (2D) PCs. When the coupling between the surface modes becomes strong, the frequency change of even mode is large but that of odd mode is slight, unlike the case of coupling between localized modes induced by point or line defects. ${ }^{16,17}$ This behavior of coupled modes can be understood from the field distribution of the modes in the PC. In addition, we show from the finitedifference time-domain (FDTD) simulations that the even mode can be used as a good guided mode.

We first consider the surface modes in 2D dielectric PCs, which are formed by a triangular array of air holes in a dielectric material. The radius of air holes and the dielectric constant of material is assumed to be $0.3 a$ and 8 , respectively, where $a$ is the lattice constant. It is well known that

\footnotetext{
${ }^{a)}$ Electronic mail: hypark@kaist.ac.kr

${ }^{b)}$ Electronic mail: cskee@gist.ac.kr
}

the PCs composed of air holes exhibit large band gaps for TE modes whose electric fields are perpendicular to the axes of air holes. Thus, we consider only TE modes in our study. Previous studies have shown that PCs can support surface modes for appropriately truncated PC surfaces. ${ }^{4-10}$

Figure 1(a) shows the top view of truncated surface of our triangular photonic crystal. The termination factor $\tau$ is defined as $\tau=x / d$ and thus describes the surface shape of the truncated PC, where $d$ is the period of the air holes of the PC along the $x$ direction. To calculate the frequencies and the field distributions of surface modes localized at the truncated surface, we employed the conjugate gradient plane-wave expansion method. ${ }^{18}$

In Fig. 1(b), the photonic bands of uncoupled surface modes are shown when $\tau$ changes from 0 to 0.4 . The straight line denotes the light line. The surface modes are very sensitive to the shape of truncated surfaces. We can see that the frequency range of surface modes cover most of the entire photonic band-gap region when $\tau$ has appropriate values between 0 and 0.1 . Note that the surface mode exists only around the edges of photonic band gap of the bulk PC when $\tau$ is between 0.2 and 0.3 .

It has been known that the characteristics of localized modes in photonic crystals are very similar to those of electron states confined in atoms. For example, a coupling between two identical localized modes makes their frequency split into a lower frequency of even mode and a higher frequency of odd mode. This phenomenon is analogous to the splitting of valence electron states in a coupled two identical atom system into the bonding and antibonding states. Two triangular PCs with the termination factor $\tau=0$ are considered to study the coupling characteristics of surface modes. The photonic bands of coupled surface modes are investigated by varying the separation distance $L$ between two truncated surfaces. We can see in Fig. 2(a) that the coupled modes split into the modes of higher and lower frequencies than that of the uncoupled mode. The coupled mode with 

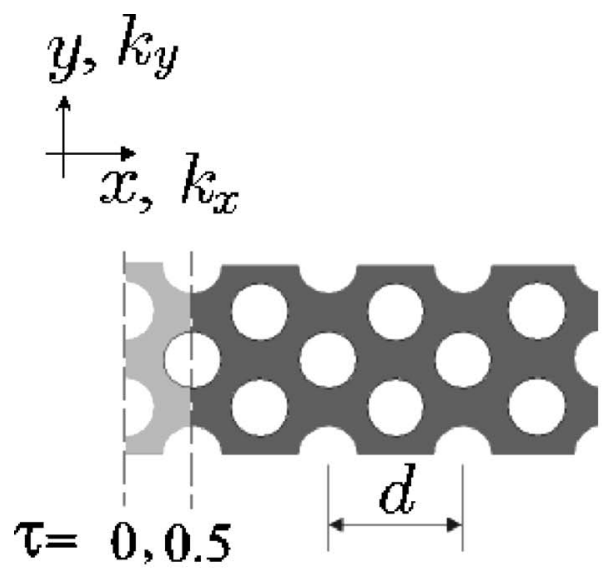

(a)

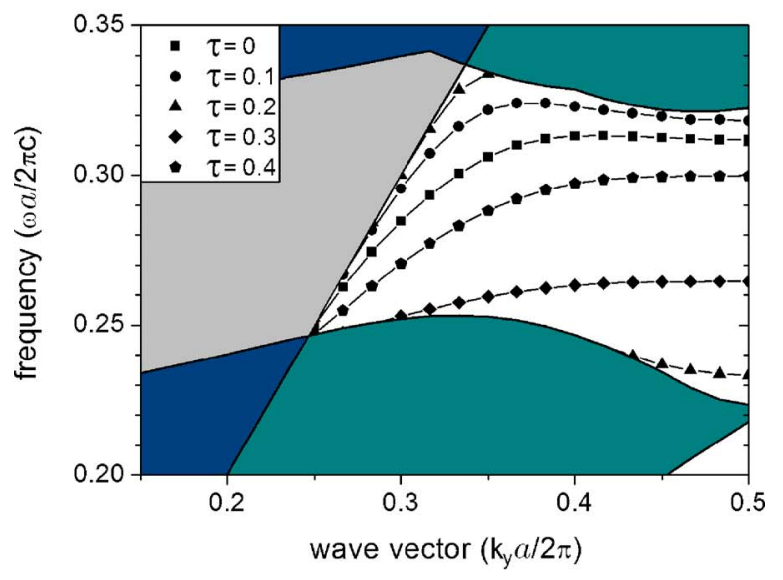

(b)

FIG. 1. (Color online) (a) The triangular lattice of air hole structure under consideration and (b) its dispersion diagram for the TE surface modes with various values of the termination factor $\tau . k_{y}=\omega / c$ defines the light line, where $k_{y}$ is the momentum parallel to the waveguide, $\omega$ the angular frequency, and $c$ the speed of light.

lower frequency has even parity and the one with higher frequency has odd parity with respect to the symmetry plane bisecting the air line between two facing PCs. The amount of splitting in frequency increases as $L$ decreases because the overlapping of the evanescent surface waves increases. It is worth noting that the frequency change of the odd mode is small as $L$ varies, whereas that of the even mode is considerable. This feature is different from the coupling characteristic of localized modes in PCs such as cavity modes created by point defects or guided modes created by line defects. ${ }^{16,17}$ In those cases, the even mode of the lower frequency and the odd mode of the higher frequency split by an equal amount with respect to the uncoupled mode.

The different behavior of two split modes can be explained in terms of their dispersion relations and field distributions. It is well known that the energy-momentum dispersion relation for $2 \mathrm{D}$ PC waveguides is given by $(\omega / c)^{2}$ $=k_{x}^{2}+k_{y}^{2}$. At a given real $k_{y}$, for a mode to be confined, $\omega / c$ should be less than $k_{y}$. As $\omega / c$ has smaller value, the imaginary part of $k_{x}\left(k_{x, i m}\right)$ becomes larger. It means that a strong field confinement occurs at lower frequencies in the $x$ direction. The penetration depths defined as $\sigma=1 / k_{x, i m}$ are about $0.716(a / 2 \pi), 0.818(a / 2 \pi)$, and $0.988(a / 2 \pi)$ for the

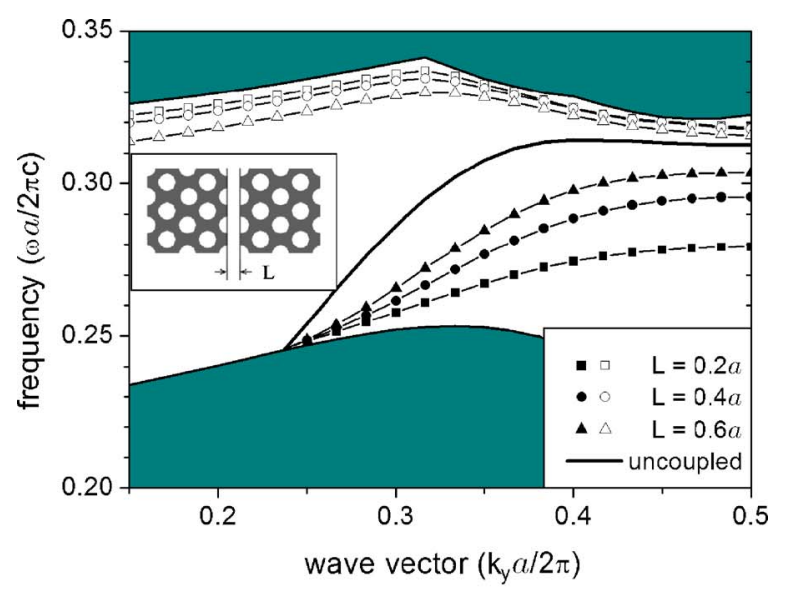

(a)

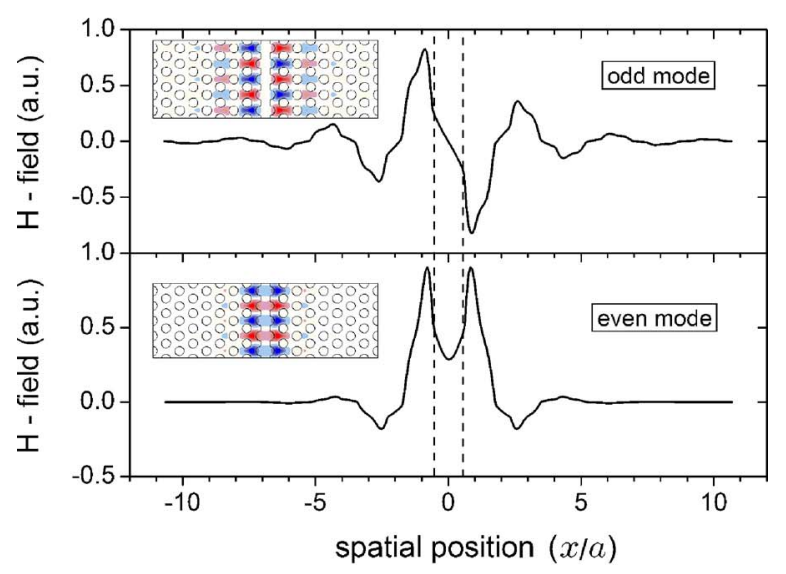

(b)

FIG. 2. (Color online) (a) The dispersion relations of the coupled and uncoupled surface modes of a triangular lattice with the termination factor $\tau$ $=0$. The inset shows the corresponding structure and the separation distance $L$ between two surfaces. Coupled odd (even) modes are denoted by open (solid) symbols. (b) The cross-sectional profiles of the magnetic field amplitudes of even and odd modes along the $x$ direction when $L=0.6 a$ and $k_{y}=0.5(2 \pi / a)$. The dashed lines indicate the positions of the surfaces. Spatial field distributions for corresponding modes are also shown in the insets.

even modes and 4.797( $a / 2 \pi), \quad 3.263(a / 2 \pi)$, and $1.942(a / 2 \pi)$ for the odd modes, respectively, for $L=0.2 a$, $0.4 a$, and $0.6 a$ when $k_{y}=0.5(2 \pi / a)$ and $\tau=0$. The penetration depth of an uncoupled surface mode is $1.441(a / 2 \pi)$. It shows that as the two truncated surfaces get closer, the confinement of the field in the direction orthogonal to the surfaces gets stronger in the even modes and weaker in the odd modes. Figure 2(b) shows the cross-sectional views of the magnetic field amplitudes of even and odd modes along the direction of $x$ when $L=0.6 a, \tau=0$, and $k_{y}=0.5(2 \pi / a)$. The dashed lines indicate the positions of the surfaces. Note that the field amplitude has its maximum not at the exact surface positions but at the centers of dielectric triangular lattices, which has been recently reported by Enoch $e t$ al. ${ }^{19}$

The differences in frequency between the even modes and the uncoupled mode $\left(\delta \omega_{e}=\omega_{0}-\omega_{e}\right)$ are $0.033(2 \pi c / a)$, $0.017(2 \pi c / a)$, and $0.009(2 \pi c / a)$ for $L=0.2 a, 0.4 a$, and $0.6 a$, respectively, whereas the corresponding values for odd modes $\left(\delta \omega_{o}=\omega_{o}-\omega_{0}\right)$ are $0.006(2 \pi c / a), 0.005(2 \pi c / a)$, $0.003(2 \pi c / a)$, where the frequency of uncoupled mode $\omega_{0}$ is 


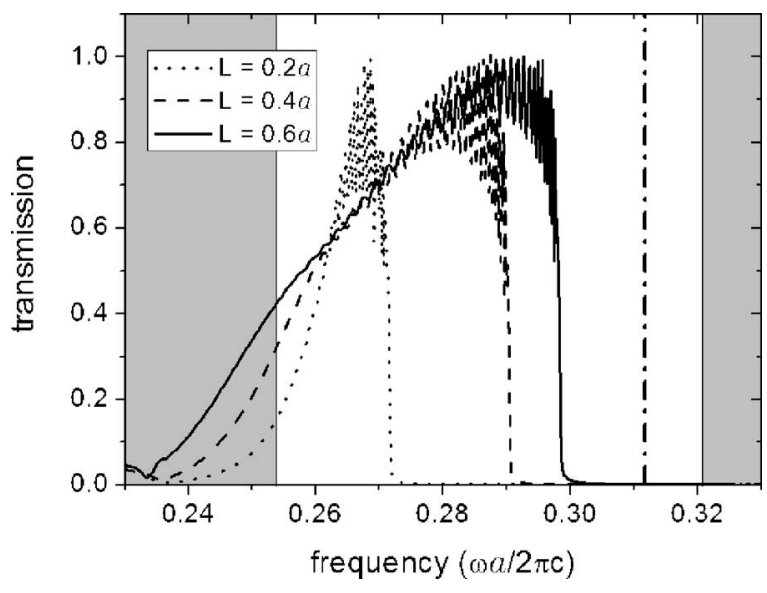

FIG. 3. Dependence of waveguide transmission for the even modes on the separation distance $L$. The dash-dotted line indicates the band edge of the uncoupled surface mode when $\tau=0$.

$0.313(2 \pi c / a)$. The amount of frequency splitting for even mode is larger than that for odd mode $\left(\delta \omega_{o}\right)$ at a given separation distance $L$. Moreover, the differences between the amounts $\left(\delta \omega_{o}-\delta \omega_{e}\right)$ get larger as $L$ decreases. The different aspects of field distribution described above in terms of the dispersion relation can explain the dependence of $\left(\delta \omega_{o}\right.$ $\left.-\delta \omega_{e}\right)$ on $L$. The fields of even modes that have lower frequencies are confined strongly around the air line between the two surfaces, but the fields of odd modes spread out widely in the PCs. Therefore, the amount of frequency splitting of odd modes is less sensitive to the separation length $L$ compared to the case of even modes.

Even modes can be used as guided modes propagating in air. Figure 3 shows the transmission spectra of even modes for $L=0.2 a, 0.4 a$, and $0.6 a$ when $\tau=0$. The transmission spectra were obtained from the FDTD simulations. We used a sufficiently large computational domain $(11 a \times 100 a)$ and perfectly-matched-layer boundary condition to remove the artifacts induced by the reflection at the crystal boundaries. The transmission ranges are in good agreement with the frequency ranges of photonic bands of even modes shown in Fig. 2(a). Since the frequencies of even modes cannot be greater than that of the uncoupled mode, $\tau$ must be carefully chosen in order to get a wide transmissive region. For example, when $\tau=0$, the band edge of the even mode, which is restricted to lie below the band of uncoupled mode, is $0.313(2 \pi c / a)$.

In Fig. 4, we show that the dispersion and transmission of the coupled even modes depend on the separation distance $W$ between two surfaces which are created by bisecting the $\mathrm{PC}$ with an air trench. Note that as the width of the air trench $W$ increases, the corresponding $\tau$ increases. Thus, the wider the air trench, the higher the frequencies of the corresponding uncoupled surface modes. For small values of the width $W$, the bands of odd modes are very close to the upper bulk band edge, since the coupling becomes strong and also the corresponding uncoupled surface modes are positioned at higher region of the band gap. This is why the odd modes are not drawn in the figure. As a result of coupling the even modes span the whole band-gap region when $W=0.6 a$, as can be seen in Fig. 4(a). The simulated results depicted in

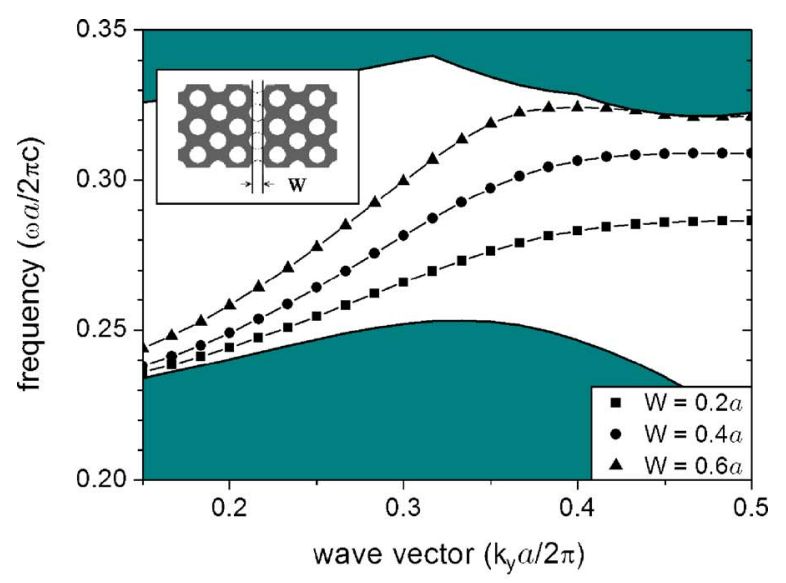

(a)

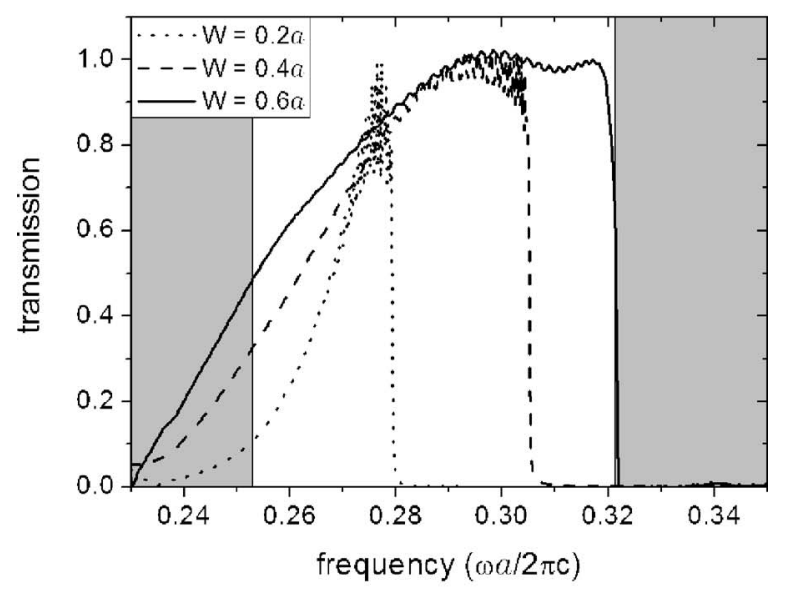

(b)

FIG. 4. (a) (Color online) The dispersion relation of the coupled modes of a triangular lattice $\mathrm{PC}$ with an air trench of width $W$. Notice that the termination factor $\tau$ alters depending on the value of $W$. (b) Dependence of waveguide transmission on the air trench width.

Fig. 4(b) show that nearly $100 \%$ transmission can be obtained for the light of frequencies ranging from $0.29(2 \pi c / a)$ to $0.32(2 \pi c / a)$ when $W=0.6 a$. Therefore, by controlling the shape and distance between the truncated surfaces of a PC, these air-trench-type waveguides can be made to possess single guide modes with a wide transmissive bandwidth and exhibit high transmission intensity as well as low group velocity.

In conclusion, we have studied the coupling properties of surface modes in the truncated 2D PC surfaces and its application to single guided modes. The confinement of fields in the direction orthogonal to the surfaces depends on the dispersion relation of the coupled modes. The difference in the amount of frequency splitting of even and odd modes with respect to the uncoupled mode is considerably large due to their different field distribution. The possibility of application of the even modes as a waveguide is also shown from the simulated results that the coupled waveguide with an air trench of $W=0.6 a$ has a very wide transmissive bandwidth. This kind of air-guided modes are very useful in the implementation for nanoscale sensing of gas or liquid, even when the amount of materials involved are ultrasmall. 
This work was partially supported by the Korea Science and Engineering Foundation through the Quantum Photonic Science Research Center.

${ }^{1}$ E. Yablonovitch, Phys. Rev. Lett. 58, 2059 (1987).

${ }^{2}$ M. Qiu, M. Mulot, M. Swillo, S. Anand, B. Jaskorzynska, A. Karlsson, M. Kamp, and A. Forchel, Appl. Phys. Lett. 83, 5121 (2003).

${ }^{3}$ S. Noda, N. Yamamoto, M. Imada, H. Kobayashi, and M. Okano, J. Lightwave Technol. 17, 1948 (1999).

${ }^{4}$ R. D. Meade, K. D. Brommer, A. M. Rappe, and J. D. Joannopoulos, Phys. Rev. B 44, 10961 (1991).

${ }^{5}$ K. W.-K. Shung and Y. C. Tsai, Phys. Rev. B 48, 11265 (1993).

${ }^{6}$ F. Ramos-Mendieta and P. Halevi, J. Opt. Soc. Am. B 14, 370 (1997).

${ }^{7}$ W. M. Robertson and M. S. May, Appl. Phys. Lett. 74, 1800 (1999).

${ }^{8}$ F. Ramos-Mendieta and P. Halevi, Phys. Rev. B 59, 15112 (1999).

${ }^{9}$ M. Qiu and S. He, Phys. Lett. A 282, 85 (2001).
${ }^{10}$ Y. A. Vlasov, N. Moll, and S. J. McNab, Opt. Lett. 29, 2175 (2004).

${ }^{11}$ R. Gonzalo, P. de Maagt, and M. Sorolla, IEEE Trans. Microwave Theory Tech. 47, 2131 (1999).

${ }^{12}$ Y.-J. Park, A. Herschlein, and W. Wiesbeck, IEEE Trans. Microwave Theory Tech. 49, 1854 (2001).

${ }^{13}$ E. Moreno, F. J. García-Vidal, and L. Martín-Moreno, Phys. Rev. B 69, 121402 (2004).

${ }^{14}$ E. Moreno, L. Martín-Moreno, and F. J. García-Vidal, Photonics Nanostruct. Fundam. Appl. 2, 97 (2004).

${ }^{15}$ J.-K. Yang, S.-H. Kim, G.-H. Kim, H.-G. Park, Y.-H. Lee, and S.-B. Kim, Appl. Phys. Lett. 84, 3016 (2004).

${ }^{16}$ C.-S. Kee, H. Lim, and J. Lee, Phys. Rev. B 67, 073103 (2003).

${ }^{17}$ F. S. Chien, Y. Hsu, W. Hsieh, and S. Cheng, Opt. Express 12, 1119 (2004).

${ }^{18}$ R. D. Meade, A. M. Rappe, K. D. Brommer, J. D. Joannopoulos, and O. L. Alerhand, Phys. Rev. B 48, 8434 (1993).

${ }^{19}$ S. Enoch, E. Popov, and N. Bonod, Phys. Rev. B 72, 155101 (2005). 